\title{
GLI ITALIANI E IL VOTO EUROPEO: MOLTE CONFERME, POCHE SMENTITE
}

\author{
di Paolo Natale
}

\section{Introduzione}

Come da molte parti è stato sottolineato, e come confermano puntualmente i sondaggi d'opinione, l'attuale fase politica nazionale è marcata da una profonda e crescente disaffezione dei cittadini nei confronti del mondo della politica in generale e di quello partitico in particolare.

Tutti i giudizi che vengono formulati dagli italiani in merito alle più rilevanti istituzioni politiche o agli attori politici occupano - nel ranking complessivo delle «fiducie» (riportate in tab. 1) - le posizioni decisamente più basse. Senza particolari distinzioni tra elettori di sinistra o di destra, la popolazione italiana appare unanime nel considerare gravemente insufficienti sia i partiti che i principali organi di rappresentanza politica.

TAB. 1. Fiducia degli italiani nelle principali istituzioni

\begin{tabular}{clcclr}
\hline Graduatoria & Istituzioni & Voto $^{a}$ & Graduatoria & Istituzioni & Voto $^{a}$ \\
\hline 1 & Polizia/CC & 7,7 & 11 & Giornali & 5,6 \\
2 & Ass. volontariato & 7,2 & 12 & Mediaset & 5,6 \\
3 & Ass. autodifesa & 6,7 & 13 & Comune & 5,4 \\
4 & Forze Armate & 6,7 & 14 & Banche & 5,3 \\
5 & Scuola & 6,6 & 15 & Regione & 5,3 \\
6 & Magistratura & 6,4 & 16 & Ass. categoria & 5,1 \\
7 & Chiesa cattolica & 6,4 & 17 & Sindacati confederali & 5,1 \\
8 & Ass. ambientaliste & 6,3 & 18 & TeleMonteCarlo & 4,9 \\
9 & Ue & 6,1 & 19 & Parlamento & 4,9 \\
10 & Rai & 6,0 & 20 & Governo & 4,9 \\
& & & 21 & Ass. imprenditori & 4,7 \\
& & & 22 & Sindacati autonomi & 4,3 \\
& & & 23 & Partiti politici & 4,2 \\
\hline
\end{tabular}

a Media dei voti da 1 a $10(1$ = poca fiducia, $10=$ molta fiducia $)$.

Fonte: Abacus, cit. in Natale (1998).

RIVISTA ITALIANA DI SCIENZA POLITICA / a. XXIX, n. 3, dicembre 1999 
Il distacco tra cittadini e istituzioni è presente in maniera diffusa e indifferenziata in tutte le aree territoriali del paese e sembra essere percepito senza particolari disomogeneità all'interno di ciascun elettorato.

Il credo politico, l'appartenenza ad uno schieramento, l'identificazione con il proprio partito di riferimento, lo stesso comportamento di voto tendono a rivestire un ruolo sempre più marginale - da parte degli elettori e dei cittadini - nella definizione della propria personalità e nella formazione dell'identità individuale e collettiva (Francioso e Natale 1992).

In occasione delle recenti elezioni europee del giugno 1999 , le conferme a questa tendenza di fondo si sono rilevate certamente più numerose delle possibili smentite. Gli elementi maggiormente illuminanti della direzione che sta prendendo il rapporto tra italiani e voto verranno evidenziati in questo breve saggio; essi sono, in particolare, quattro:

a) la crescita elettorale delle liste il cui consenso appare legato a motivazioni di voto di carattere episodico e contingente;

b) i livelli costantemente crescenti di indecisione nell'espressione del proprio orientamento di voto da parte degli elettori;

c) la quota in continua ascesa della volatilità (e infedeltà) elettorale, non solo tra una elezione e la successiva, ma addirittura nella stessa tornata elettorale, tra diversi tipi di elezione («differenziazione», secondo la definizione di Vassallo 1997, 55);

d) il costante calo della partecipazione politica visibile, prima fra tutte quella elettorale, relativa all'affluenza alle urne.

Tenendo conto di questi quattro elementi di sfondo, verranno qui analizzati - dopo un breve riassunto dei risultati di voto a livello nazionale - i maggiori flussi elettorali tra le due ultime elezioni (politiche del 1996 ed europee del 1999) e la composizione socio-demografica degli elettorati attuali dei principali partiti, in particolare delle due liste «inedite» che in questa consultazione hanno riscosso un consistente (e per taluni inaspettato) consenso elettorale: la Lista Bonino e i Democratici di Prodi. Una breve analisi dell'influenza televisiva sulla decisione di voto degli incerti chiuderà il saggio.

\section{I risultati del voto europeo.}

Per quanto possa talvolta apparire paradossale, trattandosi di dati «oggettivi», è noto che in Italia esistono da sempre mo- 
dalità molto differenti di accostarsi ai risultati elettorali di una qualsiasi consultazione: c'è chi propone confronti con le omologhe elezioni precedenti, chi utilizza come termine di paragone le ultime elezioni effettuatesi a livello nazionale, chi si basa sul confronto con le aspettative generate dai sondaggi pre-elettorali, chi ragiona in termini di coalizione, chi di lista, e così via. $\mathrm{A}$ livello di discussione politica, tutti i tipi di paragone scelti rispondono alla stretta logica di apparire in qualche modo «più vincitori» $\mathrm{o}$ «meno sconfitti» di quanto non si sia nella realtà.

Non dovendo entrare nel merito di questo tipo di dibattito politico, che si è acceso anche all'indomani delle europee, la scelta qui operata è stata quella di osservare i risultati delle ultime consultazioni dal punto di vista del comportamento elettorale degli aventi diritto al voto. Tutti i risultati saranno pertanto presentati ed analizzati in relazione all'intero corpo elettorale, e confrontati con l'ultimo appuntamento elettorale nazionale, le elezioni politiche del 1996, relativamente alla parte proporzionale. Anche in quel caso infatti, le possibili scelte dei votanti si potevano rivolgere ad un partito o ad una lista, indipendentemente dalla coalizione di riferimento.

Come si può facilmente notare nella tabella 2 , i reali «vincitori» di questa consultazione elettorale sono da ricercarsi nelle aree che maggiormente ribadiscono una relativa distanza dall'offerta politica più «tradizionale». A livello partitico, le due liste inedite dei Democratici (Prodi, Di Pietro e sindaci) e di Emma Bonino hanno complessivamente raccolto un numero totale di voti superiore ai 10 punti percentuali; a livello «a-partitico», l'area del non-voto (astensioni, schede bianche e nulle) ha raccolto numerosi nuovi adepti, con un incremento di oltre 13 punti percentuali rispetto alla precedente consultazione politica e di 6 punti rispetto alle precedenti europee del 1994.

Se teniamo poi in considerazione che Forza Italia, l'unico dei maggiori partiti che non abbia perso elettori, ha adottato una campagna legata (quasi) esclusivamente alla personalità di Berlusconi, possiamo provvisoriamente concludere che le elezioni europee del 1999 hanno visto una netta prevalenza dei «volti» (Prodi, Di Pietro, Bonino, lo stesso Berlusconi) rispetto alle issues, italiane o europee ${ }^{1}, \mathrm{e}$ - ancor più - della disaffezione partitica rispetto all'attaccamento o al coinvolgimento.

1 In un sondaggio effettuato dall'Istituto Abacus (che si ringrazia per aver messo a disposizione i dati qui presentati) nelle due settimane prima del voto, tra gli elementi 
TAB. 2. Risultati Europee 1999 e Camera 1996 (parte proporzionale) (\% sugli aventi diritto)

\begin{tabular}{|c|c|c|c|c|}
\hline Liste & 1999 & & 1996 & Differenza \\
\hline Rifond. Com. & 2,7 & ] & 6,6 & $-2,6$ \\
\hline Pdci (Cossutta) & 1,3 & ] & & \\
\hline $\mathrm{Ds}(\mathrm{Pds})$ & 11,1 & & 16,3 & $-5,2$ \\
\hline Verdi & 1,1 & & 1,9 & $-0,8$ \\
\hline I Democratici & 4,9 & & & $(+4,9)$ \\
\hline Ppi & 2,7 & & 5,2 & $-2,5$ \\
\hline Lista Dini & 0,7 & & 3,3 & $-2,6$ \\
\hline Sdi & 1,3 & & & $(+1,3)$ \\
\hline Udeur & 1,0 & ] & & \\
\hline Cdu & 1,3 & ] & 4,5 & $-0,5$ \\
\hline Ccd & 1,7 & ] & & \\
\hline Forza Italia & 16,0 & & 16,0 & $=$ \\
\hline $\mathrm{An}$ & 6,6 & & 12,1 & $-5,5$ \\
\hline Lega Nord & 2,9 & ] & 7,8 & $-4,6$ \\
\hline Liga Veneta & 0,3 & ] & & \\
\hline Lista Bonino (Pannella) & 5,4 & & 1,5 & $+3,9$ \\
\hline Ms-Fiamma Tric. & 1,0 & & 0,7 & $+0,3$ \\
\hline Altri & 1,6 & & 1,3 & $+0,3$ \\
\hline Ast. + Bianche + Nulle & 36,4 & & 22,8 & $+13,6$ \\
\hline Totale & 100,0 & & 100,0 & $=$ \\
\hline
\end{tabular}

\section{Gli italiani e l'Europa}

$\mathrm{Si}$ è sottolineato come le ultime elezioni europee abbiano enfatizzato nuove e diverse modalità di accostarsi all'appuntamento elettorale da parte degli italiani. In particolare si sono accentuati fenomeni indicativi, da una parte, di un generale maggiore rifiuto di esprimere la propria scelta partitica, dall'altra, di una estrema volatilità rispetto alle scelte effettuate nel passato, sintomo quest'ultimo di una crisi delle motivazioni le-

che l'elettore tiene in considerazione nella scelta del partito da votare, la personalità dei candidati è quello maggiormente scelto (da quasi il $40 \%$ dei 2000 intervistati). Questi i risultati completi e la formulazione originale della domanda. Nella sua scelta del partito che voterà alle europee quale dei seguenti elementi terrà più in considerazione?

- $39,5 \%$ la personalità e la competenza dei candidati

- $32,5 \%$ il modo in cui il partito vede il futuro dell'Unione europea

- $21,1 \%$ la capacità del partito di difendere i suoi interessi

- $6,9 \%$ la posizione del partito sulla guerra del Kosovo. 


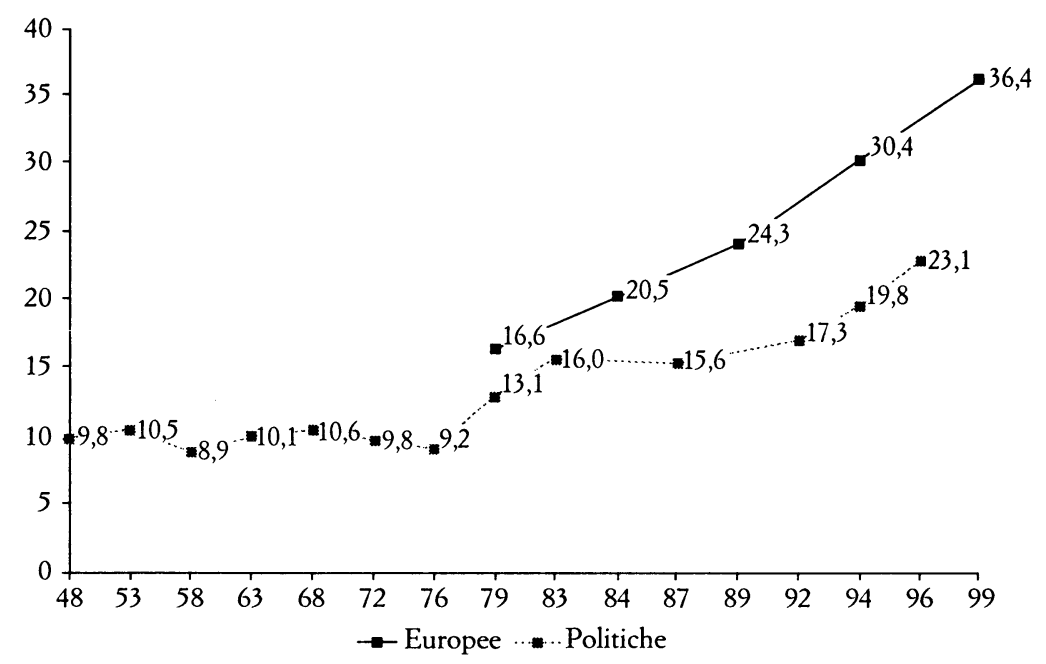

Nota: I valori sono in percentuale sul totale degli elettori.

FIG. 1. Andamento del voto non espresso (astensioni + bianche + nulle). Politiche Camera ed Europee ('48-'99).

gate alle tradizionali logiche identificative o di affinità/vicinanza per i diversi partiti ${ }^{2}$ presenti nell'arena elettorale (Pasquino 1982).

Dopo la già scarsa partecipazione all'importante referendum sul proporzionale del 18 aprile (50\% di astensioni circa), il rifiuto di partecipare alla competizione elettorale si è espresso in questa occasione attraverso un astensionismo che non ha mai avuto eguali in tutte le elezioni nazionali svoltesi in Italia $(30 \%)$, referendum esclusi. Se a questa quota sommiamo inoltre il numero di coloro che hanno scelto il voto bianco o nullo, il totale degli elettori italiani che non hanno voluto esprimere un voto per uno dei partiti scesi in campo supera il $36 \%$ degli aventi diritto, 6 punti oltre il vecchio «record» delle precedenti elezioni europee del 1994.

Nella figura 1 sono riportati i trend relativi alle elezioni legi-

2 È opportuno differenziare fin d'ora tra identificazione con un partito e identificazione con un'area politica o una coalizione. Come vedremo più avanti, lo scarso movimento inter-coalizioni sembra suggerire la presenza di inedite «affiliazioni di area», che limitano questo tipo di mobilità. 
slative ed europee, dal dopoguerra ad oggi, dell'area del «nonvoto» (astensioni + schede bianche + schede nulle). Come si può notare, l'esordio delle consultazioni europee (1979) è avvenuto in concomitanza con i primi segnali di disaffezione al voto degli italiani. Infatti, dopo un lungo periodo di stabilità sia nelle scelte di voto che nell'affluenza alle urne, dagli anni '80 in poi si assiste ad un deciso incremento dell'alterità nei confronti dei partiti tradizionali: una parte sempre maggiore dell'elettorato inizia ad esplorare nuovi percorsi e modalità di voto, ovvero accentua la propria sfiducia nella possibilità di utilizzare positivamente lo strumento elettorale, rinunciando al voto (Natale 1993, 152-155).

Per quanto riguarda le consultazioni politiche, dopo il netto aumento verificatosi a cavallo del $1980(+7 \%$ dal 1976 al 1983), la quota di astensionisti attivi o passivi non subisce ulteriori significativi incrementi fino alla fine della cosiddetta Prima Repubblica, rimanendo assestata su valori compresi tra il $15 \%$ e il $17 \%$. E soltanto nelle ultime due elezioni legislative ${ }^{3}$ che si assiste ad un nuovo deciso aumento (+6\% dal 1992 al 1996).

Viceversa, in ambito europeo la crescita continua ininterrotta, con un incremento medio del $5 \%$ tra una elezione e quella successiva. Così, il differenziale tra europee e politiche dell'area del non-voto, che era pari a circa 3 punti percentuali nel 1979, è costantemente aumentato: $+5 \%$ nel $1984,+8 \%$ nel 1989 , $+10 \%$ nel $1994,+13 \%$ nell'ultima tornata del $1999^{4}$.

Quale la causa di un così marcato disinteresse degli italiani per il Parlamento europeo? Per quanto riguardava le precedenti consultazioni, è possibile che un'Europa ancora lontana dall'essere realizzata compiutamente potesse scoraggiare la partecipazione di una quota significativa di elettori. Inoltre, il Parlamento europeo stesso non sembrava in grado, non solo agli italiani, di giocare un ruolo legislativo ed esecutivo di qualche rilievo.

3 Vassallo (1997, 46) avanza, come possibile interpretazione dell'incremento dell'astensionismo, l'accelerata frequenza delle consultazioni dal 1989 al 1996 (almeno una volta all'anno, senza contare le elezioni provinciali o comunali). Viceversa, tra le politiche del 1996 e le europee del 1999 non vi è stata alcun'altra chiamata alle urne nazionale, se si eccettuano i referendum (che hanno fatto registrare affluenze comunque molto ridotte).

4 Per il confronto politiche-europee si sono presi in considerazione gli scarti tra l'anno in cui si sono svolte le consultazioni europee e, laddove non si siano svolte politiche nello stesso anno, il valore medio dell'astensionismo tra l'elezione politica precedente e quella successiva. 
Le motivazioni per recarsi alle urne diminuivano quindi con il decrescere del voto di appartenenza o del voto «identificante» (Pizzorno 1983).

Ma alla vigilia delle ultime consultazioni lo scenario di riferimento era radicalmente cambiato: l'ingresso dell'Italia in Europa è avvenuto, come noto, con il consenso di una larghissima maggioranza dei cittadini, in tutti i settori politici e sociali. Nelle diverse tappe di avvicinamento all'unità europea economica $\mathrm{e}$ politica una percentuale sempre più elevata di italiani si è dichiarato abbastanza o molto favorevole all'unificazione.

Oggi rimane su posizioni contrarie soltanto una quota intorno al $10 \%$ della popolazione. Tale consenso generalizzato non presenta inversioni di segno nemmeno all'interno dei diversi elettorati: in tutti i partiti, i rispettivi elettori si dichiarano infatti più favorevoli che sfavorevoli all'Unione europea.

L'opzione europeista si dimostra quindi un'istanza ben accettata dagli italiani in maniera trasversale rispetto agli orientamenti di voto, sebbene il livello di fiducia nell'Europa rifletta in qualche modo «anche» le prese di posizione dei partiti di riferimento di ciascuno degli elettorati, smorzando in un caso o enfatizzando nell'altro la sostanziale accettazione generale dell'entrata dell'Italia in Europa.

Lo stesso elevato livello di fiducia viene manifestato anche nei confronti delle capacità del Parlamento europeo di incidere positivamente nella risoluzione dei problemi più avvertiti dalla popolazione: tre elettori su quattro (in numerosi sondaggi effettuati prima delle consultazioni europee) dichiaravano molta o abbastanza fiducia nel ruolo di problem solver del futuro Parlamento europeo. Anche in questo caso, esprimeva opinioni positive la maggioranza degli elettorati di tutti i partiti.

I dati qui ricordati, che evidenziano l'elevato livello di fiducia degli italiani nell'Europa e nel suo organismo di rappresentanza politica sovra-nazionale, inducono a formulare una ipotesi (per ora provvisoria, in mancanza di studi più organici su questo specifico elettorato). Essi potrebbero cioè suggerire la presenza di un corto-circuito che quasi 4 elettori su 10 hanno risolto astenendosi: in presenza di una fiducia così elevata nell' $\mathrm{Eu}$ ropa unita e nel Parlamento europeo, rinunciare a utilizzare lo strumento del voto, rinunciare a scegliere quali tra i nostri rappresentanti politici debbano rappresentarci in Europa potrebbe avere, per coloro che si sono astenuti, il significato di dubitare della volontà, o quantomeno della capacità, della nostra classe 
politica di onorare il mandato in Europa con una presenza parlamentare assidua, significativa e rilevante.

D'altra parte, quanto e come si è parlato di Europa nella campagna elettorale precedente il voto? La percezione diffusa nella popolazione era che la competizione europea non fosse altro che una carta da giocare su un differente campo di gioco, quello dell'arena politica nazionale.

Non è un caso, allora, se anche le scelte di chi si è recato alle urne abbiano privilegiato nuove proposte politiche, $\mathrm{i}$ cui leader (come Bonino e Prodi) erano già accreditati di una immagine positiva in Europa o vi si apprestavano a svolgere un ruolo essenziale.

Torna di nuovo, come elemento cardine delle motivazioni che stanno alla base della scarsa partecipazione al voto, il basso livello di fiducia nei confronti del mondo politico nel suo complesso e dei singoli partiti, percepiti non più come rappresentanti dei bisogni e degli interessi del paese, ma di quelli personali e particolaristici: un mondo a parte.

Vedremo più oltre quali tra gli elettorati delle ultime elezioni politiche abbiano fatto maggior o minor ricorso all'opzione astensionista; ma si può già fin d'ora anticipare come la disaffezione alla propria scelta precedente, in direzione dell'astensionismo passivo o attivo, appaia un fenomeno generalizzato e diffuso, trasversale a tutte le aree politiche.

\section{I flussi di voto}

Un primo riscontro della elevata «infedeltà» di voto in questi tre anni (dalle politiche del '96 alle europee del '99) si può avere attraverso l'analisi di due indicatori sintetici: l'indice di concentrazione (bipartitica) e l'indice di cambiamento', presentati in figura 2. Il primo - calcolato come semplice somma delle percentuali di voto ottenute dai due maggiori partiti - fornisce un'indicazione della tendenza alla frammentazione o alla concentrazione del sistema partitico.

Come si può notare, il livello di frammentazione delle scelte

5 I due indici corrispondono, rispettivamente, ai tradizionali indici di bipolarismo e di instabilità. Ma il primo termine, con l'avvento del nuovo sistema maggioritario, potrebbe creare confusione terminologica. Il secondo è stato oggetto di un vivace dibattito (cfr. Cartocci 1997) e giudicato da molti «parzialmente inappropriato». 


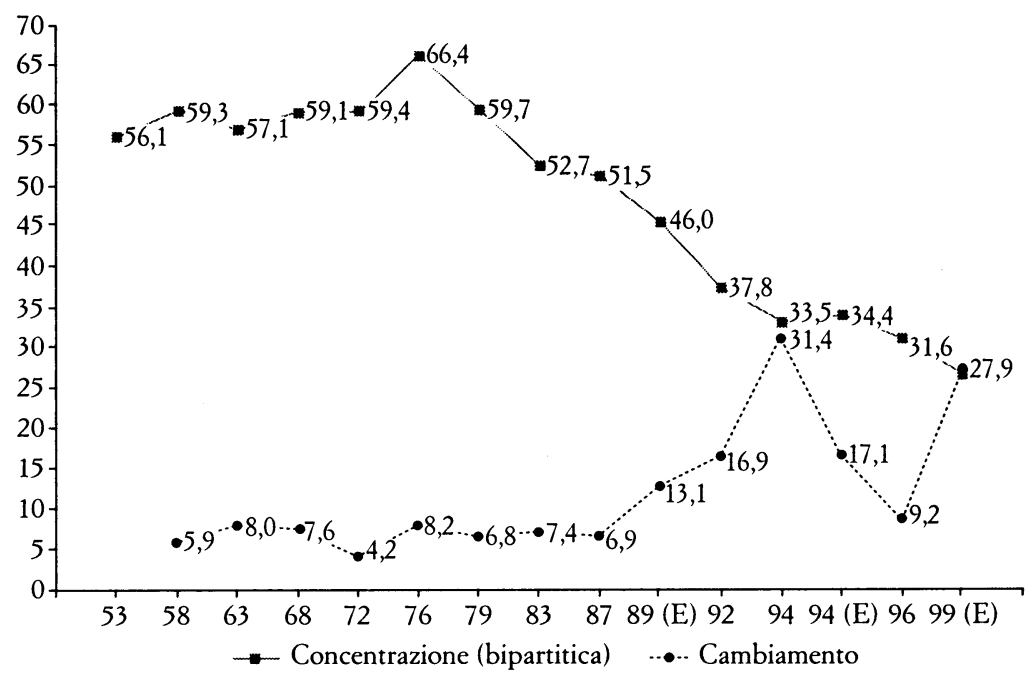

Nota: I valori sono in percentuale sul totale degli elettori.

FIG. 2. Indici di concentrazione (bipartitica) e di cambiamento. Politiche camera (prop.) + europee 89-99.

partitiche, già molto elevato nelle ultime politiche, appare ancora cresciuto. Lasciato «libero» di una scelta meno influente per il governo del paese, l'elettore manifesta (come già nel voto proporzionale delle politiche) una progressiva incapacità di concentrare le proprie preferenze su pochi partiti egemoni, facendo tendenzialmente aumentare - ad eccezione del caso di Forza Italia - il peso delle liste subalterne all'interno delle due principali coalizioni.

Oggi, poco più di un quarto dell'elettorato italiano si concentra sui due partiti maggiori (Forza Italia e Ds); soltanto vent'anni fa, tale quota superava il $60 \%$ (Dc e Pci). E la stessa somma dei primi tre partiti (includendo $\mathrm{An}$ ) riesce soltanto a eguagliare il livello di concentrazione delle ultime elezioni politiche, di poco superiore al $30 \%$ degli aventi diritto. L'apparente paradosso che demarca il rapporto tra gli italiani ed il voto viene di nuovo confermato: mentre il sistema elettorale adottato in molte consultazioni - e le varie proposte in esame in Parlamento - tende a semplificare il quadro politico-elettorale di riferimento in senso bipolare o tripolare, le scelte degli elettori relative alla tradizionale logica del partito più vicino o affine (il 
cosiddetto «voto del cuore») sembrano procedere in senso opposto (Natale 1997).

Nella figura 2 è illustrato anche il trend dell'indice di cambiamento che, calcolato come semisomma delle differenze percentuali tra i risultati degli schieramenti in campo (partiti e area del non-voto) tra una elezione e la precedente, può essere inteso quale misura della variazione complessiva dei rapporti di forza elettorali registratasi nelle tornate elettorali considerate.

Come noto, è questo il primo elemento che può darci una parziale indicazione del livello di riconferma delle scelte elettorali. Essendo calcolato a partire dai saldi di voto ai partiti o all'astensionismo, l'indice di cambiamento nasconde infatti quei mutamenti di voto che, avendo quote simili in relazione ai due medesimi referenti (partito di entrata e di uscita), si annullano a vicenda. $\dot{E}$ comunque un dato che ci indica il livello minimo possibile della fedeltà alle opzioni passate.

In questa consultazione, anche procedendo ad alcuni «accorpamenti di area», il livello di cambiamento elettorale appare ritornare elevatissimo (prossima al 25\%), vicino ai valori del 1994, quando peraltro la drastica trasformazione dell'offerta politica (in occasione del post-Tangentopoli) non poteva che provocare un forzato mutamento di opzione elettorale $e^{6}$.

Si è detto che, così calcolata, questa può essere considerata la soglia minima della mobilità globale. Attraverso l'analisi dei flussi elettorali è possibile viceversa stimare la fluidità complessiva della totalità dell'elettorato.

Seppure con maggiore difficoltà del consueto $^{7}$, anche in questa occasione elettorale è possibile verificare l'entità e la di-

6 A causa delle diverse frammentazioni interne che hanno visto protagonisti alcuni partiti dal 1996 ad oggi, il saldo è stato calcolato riaccorpando le nuove sigle. Così, ad esempio le varie Leghe (Lega nord, Liga veneta, ecc.) sono state considerate come un'unica lista, così tutti i partiti minori (sotto la voce «altri»). Nella realtà, quindi, il valore dell'indice di cambiamento sarebbe ancora maggiore di quanto riportato.

7 I comuni italiani (in ossequio al Decreto ministeriale 2 aprile 1998, n. 117), allo scopo di contenere $\mathrm{i}$ costi che vengono sostenuti in occasione delle consultazioni elettorali e per ridurre di circa il $30 \%$ il numero complessivo di sezioni elettorali, hanno adottato, entro la fine del 1998, i nuovi limiti costituiti da un minimo di 500 e un massimo di 1.200 elettori per sezione, mediante ridisegno delle sezioni medesime. Sia per le proiezioni elettorali che per l'analisi dei flussi, questa riorganizzazione ha comportato la scelta di circa 950 sezioni elettorali che non avessero sostanzialmente mutato, pur accorpandosi, i confini che avevano nella consultazione elettorale precedente, quella delle politiche del 1996. L'universo di riferimento attuale è costituito da circa 63.000 sezioni elettorali, così come sono emerse da tale menzionato ridisegno. Il campione delle 950 sezioni era distribuito in circa 460 comuni. 
rezione che hanno preso i mutamenti di voto, facendo come sempre ricorso a due tipi di fonti ${ }^{8}$ : le dichiarazioni di voto fornite dagli intervistati subito dopo essersi recati alle urne ${ }^{9}$ e l'analisi basata sulle sezioni di voto, mediante il cosiddetto «modello di Goodman», utilizzando la tecnica dei flussi elettorali a livello aggregato ${ }^{10}$, a partire dalle circa 950 sezioni-campione utilizzate da Abacus per le proiezioni elettorali.

Lo studio dei flussi elettorali effettuato attraverso questo secondo metodo ha il vantaggio di tenere sotto controllo l'intero universo degli elettori; si rende conto in tal modo anche della scelta del non-voto (astensioni, schede bianche e nulle) che, premiando o punendo in maniera talvolta differenziata le forze politiche in campo, può giungere a determinare gli esiti del confronto.

Nella tabella 3 sono mostrati i principali flussi di voto dalle politiche 1996 (parte proporzionale) alle europee del 1999, calcolati in percentuale sul totale degli elettori. Per comodità di lettura, sono state accorpate ex-post le liste minori appartenenti alla stessa «area politica» (sotto la voce «altri di centro-sinistra», ecc).

In questa tornata la quota di coloro che hanno mutato la propria preferenza di voto si situa su valori vicini al $45 \%$ dell'intero corpo elettorale (ivi compresi astensionismo e voti non validi). Anche in questo caso si notano elementi di discontinuità

8 Per l'analisi individuale, si sono utilizzate le 4000 interviste telefoniche «postvoto" realizzate da Abacus per conto di Rai e Mediaset; per l'analisi aggregata, i risultati delle 950 sezioni-campione utilizzate da Abacus per le proiezioni elettorali nella stessa serata.

9 Per non appesantire ulteriormente la lettura, non viene qui presentata anche la tabella relativa ai flussi di voto derivanti dalle interviste. I risultati, che sono peraltro a disposizione di chi fosse interessato a consultarli, non si discostano in maniera significativa - fatta salva la differente modalità di rilevazione - da quelli delle sezioni.

10 Come ormai noto, il modello, originariamente sviluppato dal sociologo americano Leo Goodman in termini statistici generali, è stato perfezionato e applicato allo studio del comportamento elettorale dall'Istituto Cattaneo di Bologna (in particolare da Hans Schadee). L'attendibilità dei risultati ottenuti con questo metodo è stata ampiamente dibattuta in Italia dagli studiosi del comportamento elettorale (per un'analisi specifica, si vedano Schadee e Corbetta 1984; Biorcio e Natale 1987; 1989; e il volume dei «Quaderni della Fondazione Feltrinelli» n. 44 del 1993, che presenta tutte le «voci» della discussione metodologica in merito al modello). L'applicazione del modello - che utilizza i dati raccolti per sezione elettorale - ha differenti possibili ambiti di riferimento territoriale, a partire da quello comunale (o sub-comunale) fino a giungere a quello nazionale. Le tecniche per una sua corretta utilizzazione si basano su alcuni complessi algoritmi matematici, che hanno come principio-guida il metodo di disaggregazione tramite regressione (disaggregation by regression). Essi permettono di fornire la stima più attendibile dei reali flussi di voto registratisi tra le due elezioni prese in esame, ivi compresi i passaggi tra il voto e l'astensionismo o viceversa. 


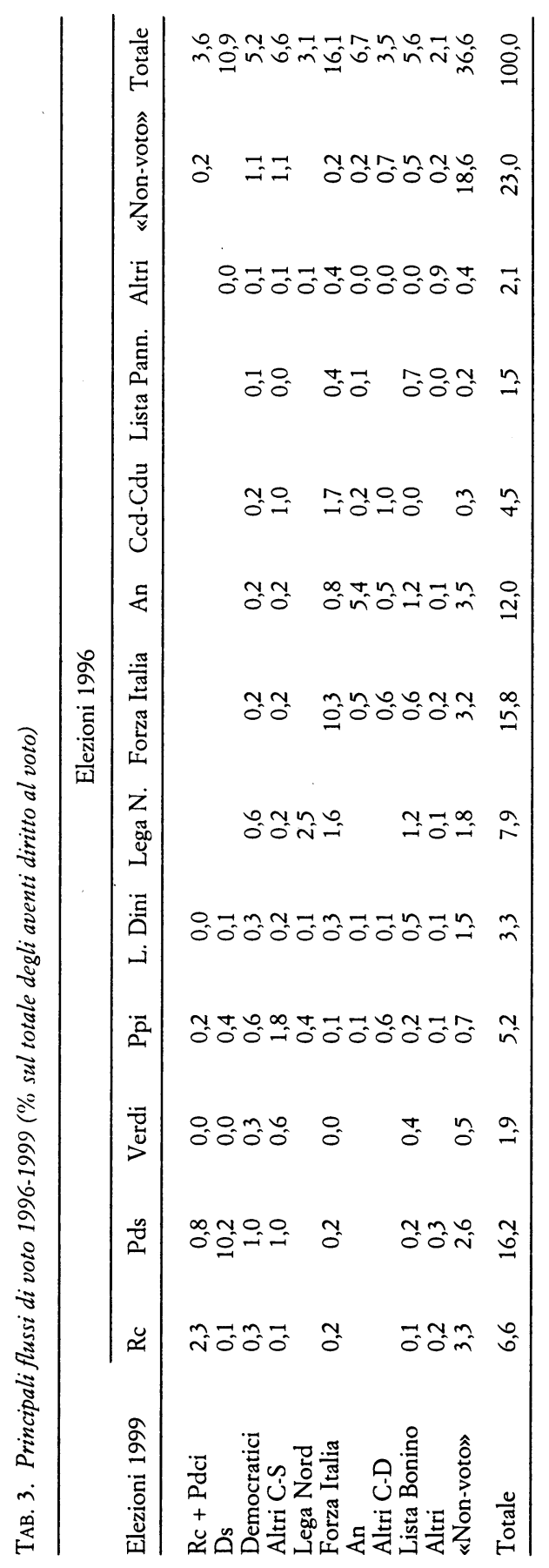


con il passato: il dato appare infatti molto più elevato rispetto a quello tradizionalmente registrato nel corso degli anni ' 80 e nel passaggio '94-'96, quando l'indice di fluidità restava compreso tra il $30 \%$ e il $40 \%$ degli elettori.

Ma dall'analisi dei flussi di voto è possibile evidenziare viceversa anche un elemento di continuità con il passato: la relativa impermeabilità delle due maggiori coalizioni o, se così si può chiamarla, la tenuta delle «nuove famiglie politiche».

In riferimento agli anni del secondo dopoguerra, la letteratura politologica aveva spesso assegnato un peso determinante nelle scelte di mutamento di voto alla logica dei blocchi o alla contiguità politica delle forze in competizione. Secondo tale interpretazione, gli elettori mobili si sarebbero spostati più facilmente o all'interno dello stesso blocco ovvero in direzione del partito giudicato limitrofo sull'asse destra-sinistra (Pasquino 1982, 138).

E anche gli studi empirici confermavano tale interpretazio$n e^{11}$ : almeno fino alle elezioni del 1983 , sia le analisi basate su dati di sondaggio sia quelle basate su dati aggregati mostravano infatti come la quota dei movimenti elettorali all'interno dei blocchi politici fosse sempre nettamente superiore a quella tra i blocchi. Gran parte della mobilità si rivolgeva, come punto di arrivo, alle forze in competizione politicamente contigue.

Già nel passaggio 1994-96 si manifestava un fenomeno dello stesso tipo: il numero degli elettori che cambiano voto restando all'interno della stessa coalizione (di centro-sinistra o di centrodestra) è stimabile in circa il doppio del numero di coloro che passano ad un partito di una coalizione diversa dall'originaria, ivi compresa la Lega Nord (Natale 1997).

Anche in questa occasione, pur in presenza di un movimento che coinvolge quasi la metà dell'elettorato e dell'incremento dell'offerta partitica, la quota di coloro che passa da un partito della coalizione di centro-sinistra (e Rifondazione comunista) ad uno della coalizione di centro-destra - o viceversa - è stimabile in poco più del $3 \%$ dell'intero elettorato, meno di un decimo del movimento complessivo fatto registrare tra le due consultazioni.

Le tabelle 4 e 5 ripropongono gli stessi dati dei flussi, calco-

11 Dalla metà degli anni '80 alle elezioni del 1992, in concomitanza con il progressivo spostamento di posizione politica del Psi e la crescita leghista, il tradizionale modo di accostarsi allo spazio politico aveva invece perso gran parte delle sue capacità descrittive e analitiche (Mannheimer e Sani 1988; Natale 1993). 


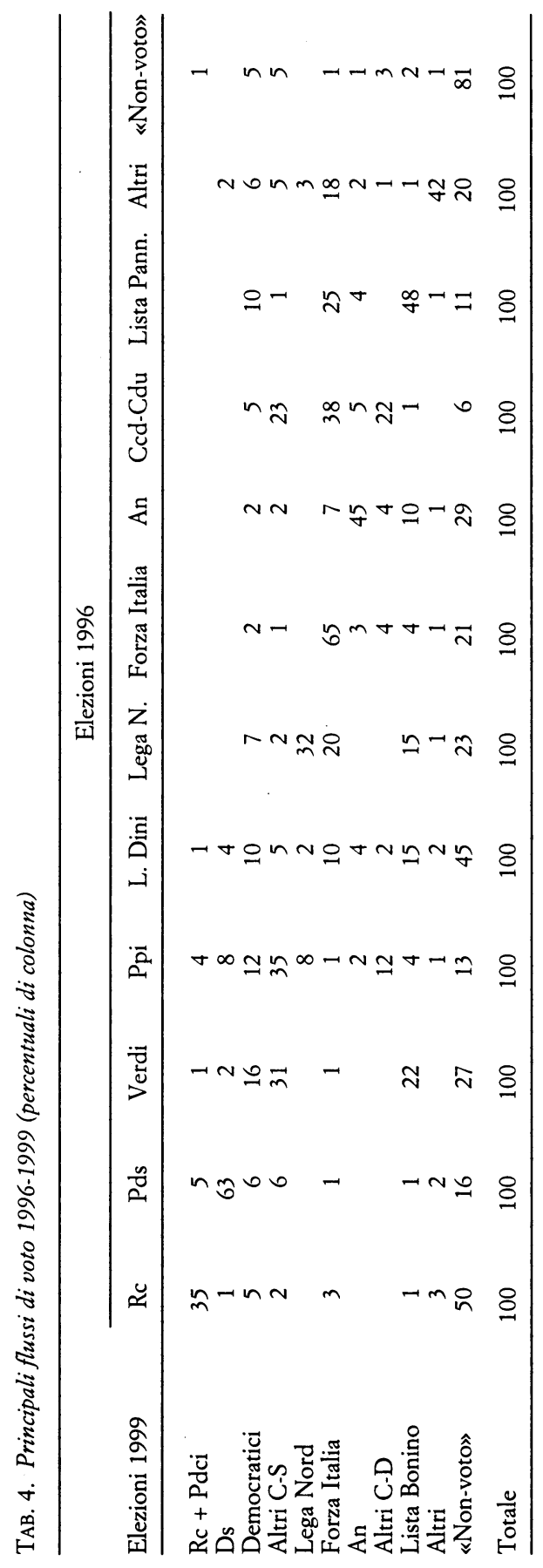




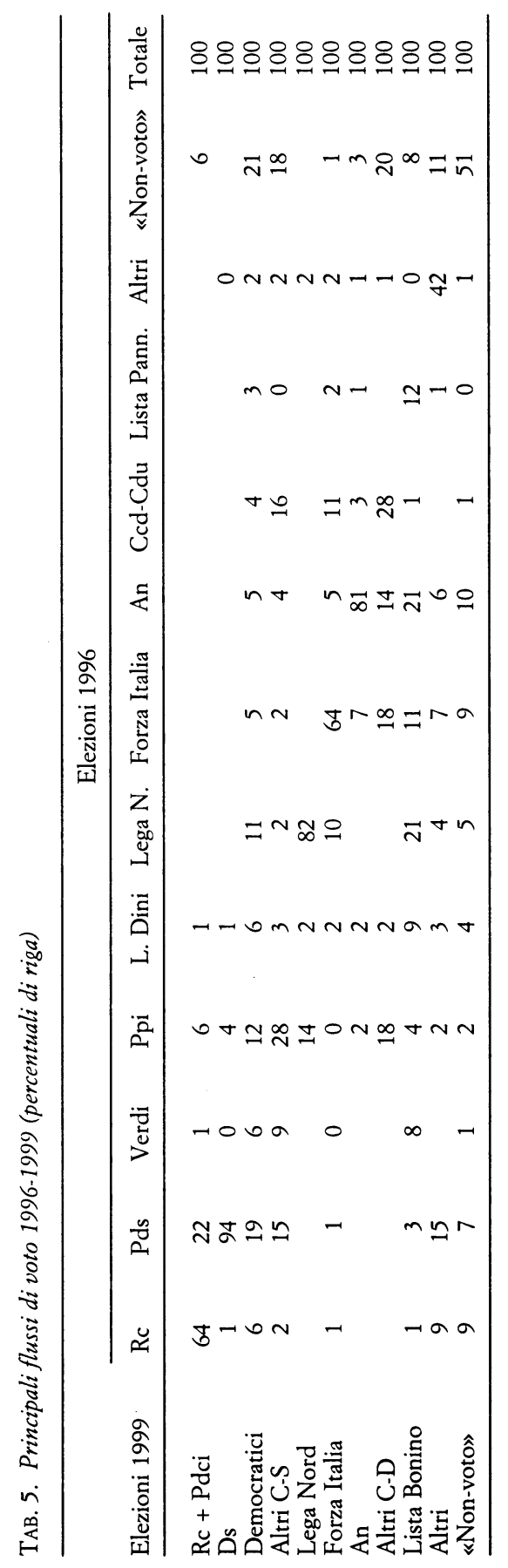


lati questa volta in percentuale sull'elettorato 1996 e sull'elettorato 1999 . La tabella 4 ci dice in pratica qual è stato il comportamento di voto degli elettori del 1996 di ognuno dei principali partiti; la tabella 5 fornisce, viceversa, indicazioni sulla composizione dell'elettorato attuale in funzione del voto precedente. Quest'ultima tabella appare di particolare interesse per quanto riguarda soprattutto i due nuovi raggruppamenti dei Democratici e della Lista Bonino. Vediamo in sintesi quali sono i cinque punti salienti che hanno demarcato, a livello elettorale, le consultazioni europee del 14 giugno:

A - Il voto per la Lista Emma Bonino. Si è trattato di un voto chiaramente legato alla contingenza: nei sondaggi immediatamente post-elettorali soltanto la metà degli elettori di questa lista ribadisce infatti l'intenzione di fidelizzare la propria scelta anche nelle prossime consultazioni. L'elettorato della Lista Bonino sembra provenire da quasi tutte le aree politiche: $25 \%$ dalla sinistra, $20 \%$ dalla Lega, $30 \%$ dalla destra, $15 \%$ dalla Lista Pannella e $10 \%$ dall'area del non voto. Si tratta quindi di un voto trasversale, in parte anti-partitico, e basato sull'appeal specifico del Commissario europeo uscente. I sostenitori di questa lista sono in particolare donne, giovani e con scolarizzazione medio-alta (tab. 6).

$B$ - Il rafforzamento di Forza Italia a scapito di An e Ccd. Il movimento di Berlusconi è uscito vincente nello scontro con quello di Fini, che ne minacciava l'egemonia, contando su una consistente quota di fedeli (due terzi del proprio elettorato del 1996), su un marcato appoggio dell'ex-elettorato leghista (oltre il $20 \%$ ) e sulla diaspora del vecchio elettorato Ccd-Cdu (quasi il $40 \%$ ha infatti scelto Forza Italia).

C - La sconfitta della Lega di Bossi. Segnali di drastica riduzione dell'attrazione leghista si erano già avuti nel corso dei primi mesi del 1999: la crisi di rappresentanza in Veneto (con la nascita della nuova Liga veneta, peraltro poco gratificata dalle scelte degli elettori), parole d'ordine sempre più sfuocate, un appoggio contraddittorio alla Serbia, ecc. Nelle europee soltanto un terzo degli elettori leghisti del 1996 ha confermato la propria scelta di voto. Gli altri hanno percorso diverse direzioni: il $10 \%$ verso il centro-sinistra (in particolare verso i Democratici), il $20 \%$ verso Forza Italia, come ricordato, il $15 \%$ verso la Lista Bonino e il $25 \%$ verso l'astensionismo.

$D$ - Il calo dei 4 maggiori partiti di governo (Ds, Ppi, Verdi e Dini). Tutti questi partiti hanno sofferto una crisi elettorale 


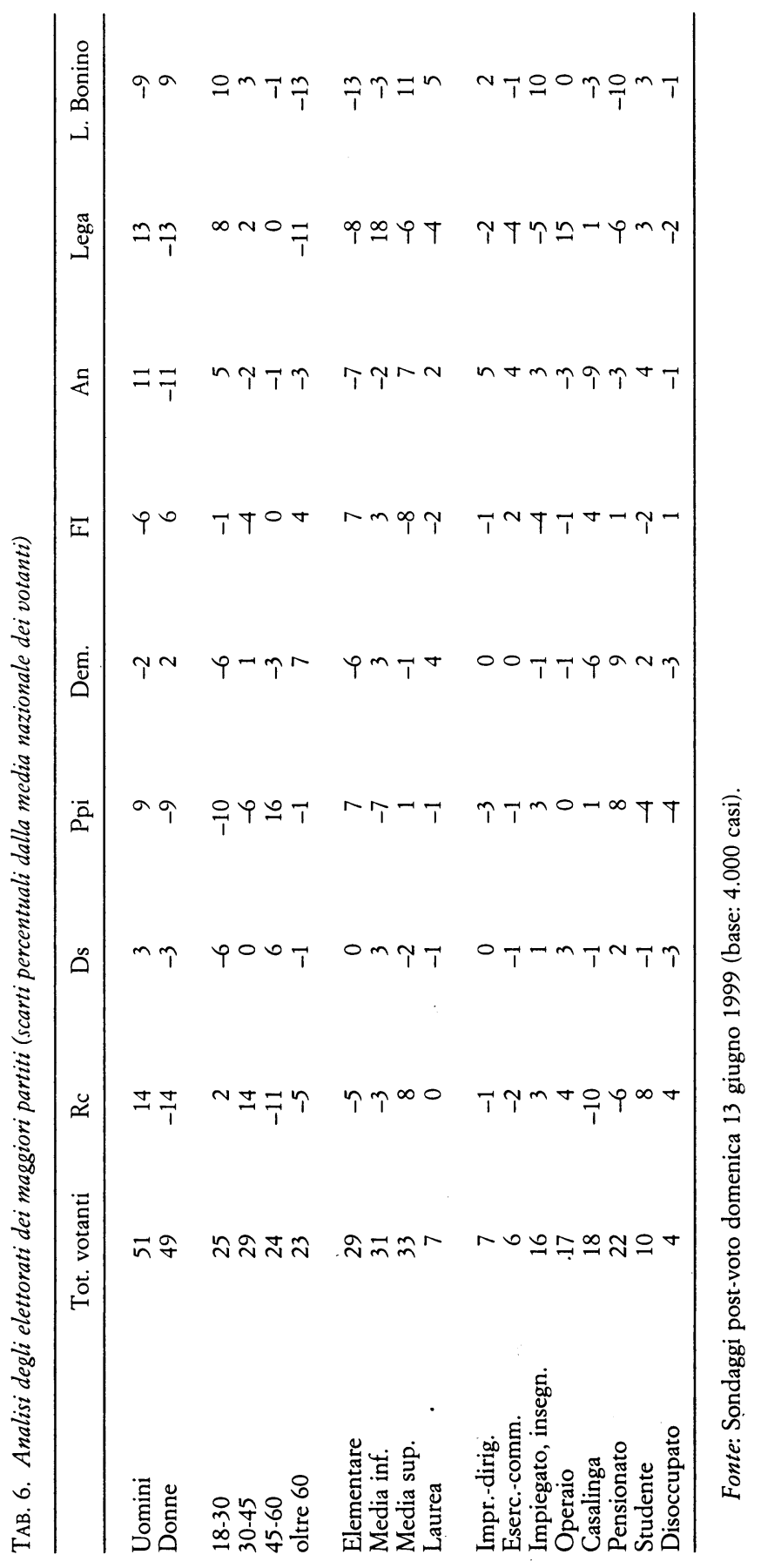




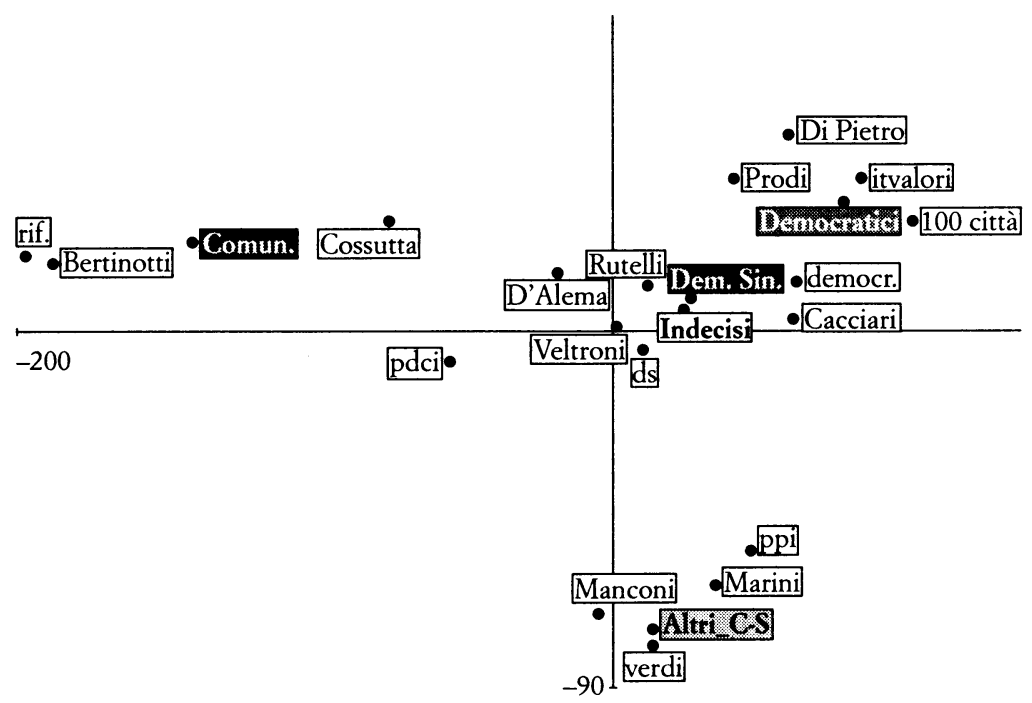

FIG. 3. Mappa elettorato votanti Ulivo 1996.

Fonte: Abacus. Base: campione di 1.003 intervistati - elettori Ulivo (maggio 1999).

considerevole: in particolare Popolari, Diniani e Verdi hanno fatto riscontrare una fedeltà elettorale inferiore al 30\% del proprio elettorato precedente, riducendo ai minimi termini la percentuale di adesione. Tutti insieme, i partiti minori del centrosinistra, riescono infatti a stento a raggiungere la percentuale ottenuta dai Democratici. Per quanto riguarda i Ds, la fedeltà degli elettori è stata in questa occasione abbastanza bassa rispetto agli anni passati (meno di due terzi del suo precedente elettorato). Ma la ragione prima del loro arretramento sembra essere legata soprattutto al mancato appeal presso altre fasce di elettorato: la quota di «ricambio» elettorale è infatti vicina allo zero (poco più del $5 \%$ ). I pidiessini «infedeli» hanno comunque privilegiato la scelta di altri partiti del centro-sinistra (quasi il $20 \%$ ) e l'area dell'astensionismo (oltre il $15 \%$ ).

E - Il successo dei Democratici di Prodi. Benché sovrastimata nei mesi precedenti il voto, la quota di elettori che hanno scelto i Democratici è risultata infine consistente: essi provengono per quasi la metà da partiti della sinistra o del centro-sinistra (in particolare $20 \%$ dal Pds e $12 \%$ dal Ppi) ma, al contrario delle aspettative, anche da altri elettorati (10\% dalla Lega, 15\% dal 
centro-destra e oltre il $20 \%$ da ex-astensionisti). Uno studio di posizionamento dell'elettorato che nel 1996 aveva votato per il centro-sinistra (Ulivo + Rifondazione comunista), presentato nella figura 3, rende evidente l'estrema contiguità tra gli elettori dei Ds e dei Democratici di Prodi. La mappa è stata costruita a partire dalle dichiarazioni di vicinanza/lontananza di ogni intervistato con i maggiori leader delle diverse liste e con i diversi partiti nel loro complesso. Abbiamo visto nello studio dei flussi elettorali come la provenienza dell'elettorato dei Democratici sia estremamente variegata, con quote soltanto minoritarie dal Pds. Nonostante ciò, questo elettorato si posiziona nel medesimo «quadrante» di quello dei Democratici di Sinistra: i giudizi di questi due elettorati - unitamente a quello degli indecisi appaiono particolarmente simili.

\section{L'influenza televisiva sul voto degli incerti}

Accanto all'elevata quota di astensionisti attivi o passivi, in questa consultazione elettorale si è assistito ad un ulteriore fenomeno inedito - quantomeno per la proporzione che ha raggiunto: l'estrema indecisione su quale partito scegliere che ha caratterizzato l'elettorato italiano.

Come si può notare dai dati presentati nella tabella 7 , tre settimane prima del voto quasi due terzi degli intervistati, tra quelli che dichiaravano di essere intenzionati ad andare a votare, non aveva ancora deciso a quale partito destinare il proprio voto. Tale quota cala ovviamente nelle settimane successive, ma resta impressionante il dato che riguarda l'ultima settimana: le interviste compiute tre giorni prima della consultazione indicano una quota di elettori incerti ancora superiore al $40 \%$.

Sebbene anche nel recente passato si sia assistito ad un cre-

TAB. 7. Quota di indecisi per settimana di rilevazione

\begin{tabular}{lcccc}
\hline Settimane prima della consultazione & 3 & 2 & 1 & 0 \\
\hline Ha già deciso per che partito votare & $37 \%$ & $38 \%$ & $49 \%$ & $59 \%$ \\
Non ha deciso per che partito votare & $63 \%$ & $62 \%$ & $51 \%$ & $41 \%$ \\
\hline
\end{tabular}

Fonte: Abacus (interviste telefoniche a 2.000 elettori che andranno a votare per le europee). 


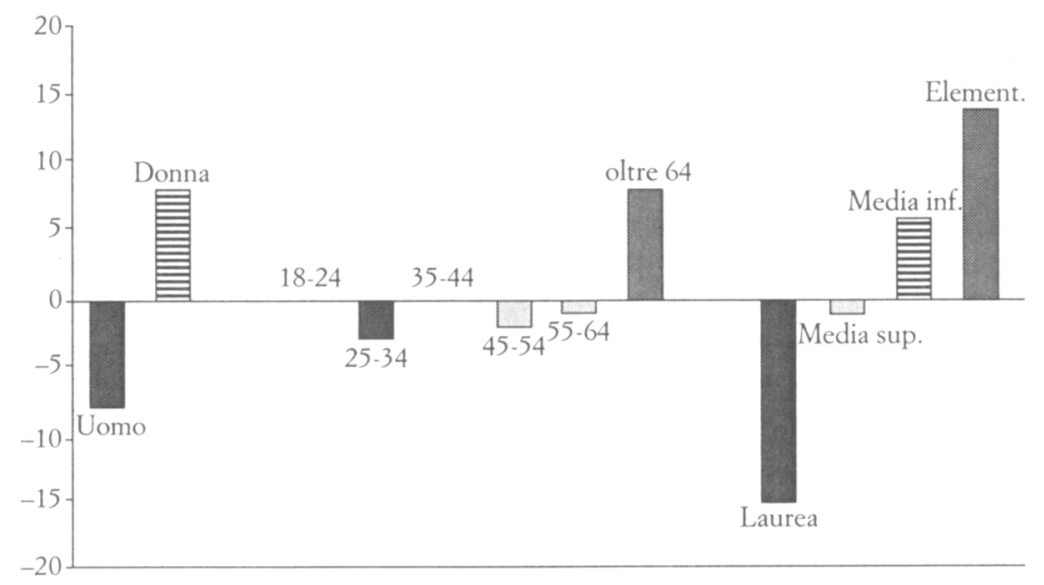

FIG. 4. Analisi caratteristiche socio-demografiche elettori indecisi (scarti dalla media).

Fonte: Abacus: sondaggi pre-elettorali maggio 1999 (2.000 interviste telefoniche nazionali).

scente fenomeno di indecisione, in particolare per quanto riguarda il voto di lista (molto meno viceversa per il voto di coalizione), mai si era registrato un livello di aleatorietà così elevato nei confronti del proprio possibile partito di riferimento.

$\mathrm{Ma}$ chi sono questi indecisi, qual è il loro livello di informazione politica e, soprattutto, a quali strumenti si affidano per poter giungere alla fine alla propria scelta elettorale? In una elezione caratterizzata da diverse polemiche sul ruolo giocato dagli spot e dalla televisione, è certamente importante comprendere se e in che misura gli indecisi si «affidino» all'informazione o quantomeno alla fruizione televisiva per poter operare la scelta di voto.

Per compiere questa analisi, il campione di intervistati è stato suddiviso nei due gruppi emersi dalla domanda sulla decisione di voto: rispetto alla media delle settimane di rilevazione, gli indecisi rappresentano quindi il 52\% del campione, i decisi il restante $48 \%$.

Vediamo innanzitutto (fig. 4) le caratteristiche socio-demografiche degli indecisi. Come si può notare, essi sono mediamente sovra-rappresentati nelle cosiddette fasce «non centrali» della popolazione elettorale: donne, anziani e livello di istruzione elementare. Gli indecisi hanno inoltre un livello di informazione 
TAB. 8. Percentuale di intervistati che dichiara «mai o quasi mai»

\begin{tabular}{lll}
\hline & Decisi & Indecisi \\
\hline Leggono articoli di politica & $23 \%$ & $68 \%$ \\
Seguono dibattiti politici in Tv & $21 \%$ & $70 \%$ \\
Discutono di politica & $22 \%$ & $69 \%$ \\
\hline
\end{tabular}

Fonte: Abacus (interviste telefoniche a 2.000 elettori che andranno a votare per le europee).

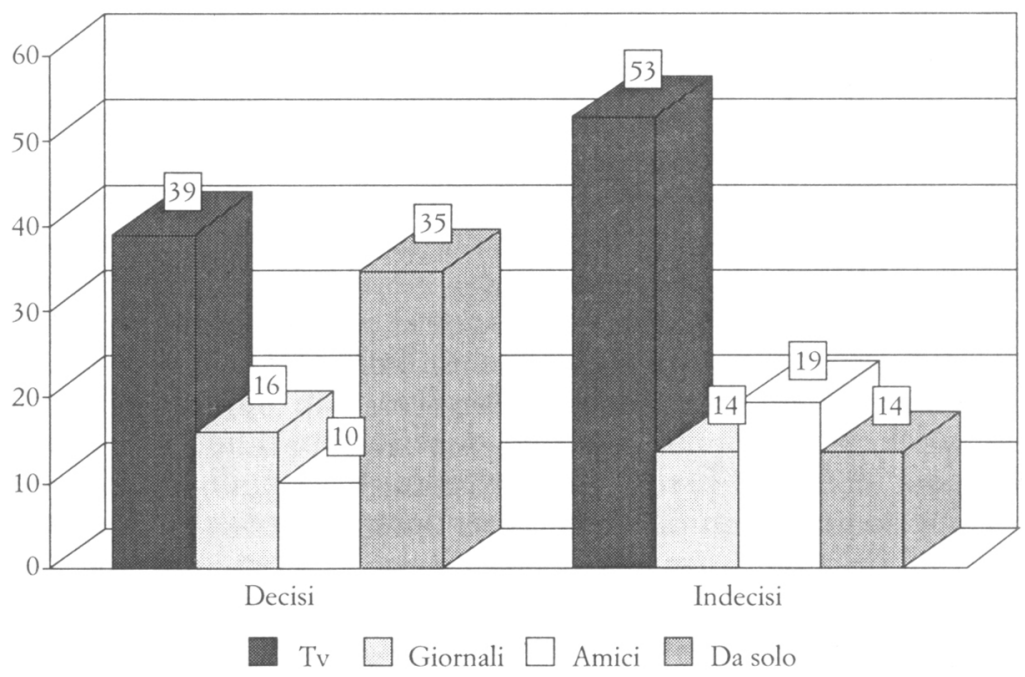

FIG. 5. Decisione di voto per canale utilizzato per informarsi.

Fonte: Abacus: sondaggi pre-elettorali maggio 1999 (2.000 interviste telefoniche nazionali).

politica nettamente meno elevato di coloro che hanno già deciso cosa votare (tab. 8): sono pertanto più «condizionabili» dai messaggi che giungono loro nelle settimane precedenti il voto.

Come pervengono, infine, alla decisione di voto, quali canali utilizzano per informarsi sui candidati o sui partiti in lizza? Come si vede chiaramente dalla figura 5 , il canale privilegiato per farsi un'idea dell'offerta politica è rappresentato per il $53 \%$ degli indecisi dalla Tv (contro soltanto il 35\% dei decisi, i quali si affidano prevalentemente alle proprie autonome capacità di scelta e di giudizio). 
Questo non significa tout court che siamo in presenza di una situazione di condizionamento televisivo del voto. $\mathrm{Ma}$ a fronte di un livello culturale e informativo decisamente ridotto da parte della quota di popolazione elettorale indecisa, che difetta quindi di strumenti idonei per discernere il tipo di messaggio ricevuto via etere, la semplificazione comunicativa cui spesso alcuni partiti si affidano rende probabilmente più facile una scelta, meno «ponderata», in quella specifica direzione.

\section{Conclusioni}

Le analisi che si sono qui presentate hanno mostrato risultati che permettono di rispondere, almeno in via provvisoria, ad alcuni dei quesiti che ci eravamo posti in sede introduttiva, e che concernono in nuce il differente rapporto che si sta instaurando oggi tra cittadini e politica, tra elettori e consultazioni elettorali.

I quattro elementi maggiormente illuminanti della direzione che sta prendendo tale rapporto sono stati approfonditi alla luce dei comportamenti adottati dagli italiani durante le ultime elezioni europee. Essi ci permettono, se non di anticipare i comportamenti futuri, di comprendere almeno alcuni aspetti cruciali della tendenza elettorale in Italia. E saranno presumibilmente questi stessi elementi a fornire le basi per le scelte che gli elettori compiranno nei prossimi anni di vita politico-elettorale.

In occasione delle ultime elezioni politiche, si evidenziava come fosse ancora difficile, o prematuro, riuscire a trovare la «cifra» precipua di quelle consultazioni: elementi di continuità con il passato si miscelavano infatti con elementi di radicale mutamento.

Oggi, a tre anni di distanza e con un tipo di elezione meno importante per l'arena nazionale e meno coinvolgente per l'elettorato, possiamo viceversa sviluppare argomentazioni più solide e sistematiche, che riguardano da una parte le logiche e le scelte di voto, dall'altro il rapporto più generale con la politica.

Dal punto di vista delle logiche di voto, l'apprendimento da parte degli elettori delle caratteristiche del nuovo sistema elettorale sembra condurli verso un utilizzo più articolato delle risorse a loro disposizione per incidere sullo scenario politico. Dal 1993 ad oggi, si sono succeduti almeno cinque appuntamenti elettorali (politici e amministrativi) in cui il cittadino ha potuto 
sperimentare un duplice voto ${ }^{12}$ sincronico (maggioritario e proporzionale) e diacronico (primo e secondo turno).

Attraverso il duplice voto ai cittadini viene fornita la possibilità di accostarsi alla competizione secondo modalità inedite, che fanno riferimento a due «percorsi» in parte contrapposti. Nel 1996 si argomentava come il voto proporzionale potesse essere identificato come una sorta di «voto del cuore», laddove il maggioritario derivasse da una scelta più razionale (Natale 1997, 208).

A fronte della ricordata consuetudine del doppio voto da una parte e della progressiva erosione dell'elettorato di appartenenza partitica, oggi i due approcci sembrano potersi ribaltare. L'elettore pare infatti aver maturato una sorta di identificazione più con la coalizione di riferimento che con uno specifico partito.

Secondo questa interpretazione, appare allora più facile «tradire» il partito che non «tradire» la coalizione; l'abbandono della propria coalizione avviene non tanto in direzione del polo contrapposto (tra centro-sinistra e centro-destra), quanto di scelte intermedie, non impegnative, episodiche (l'astensionismo, ad esempio, o liste a carattere congiunturale). Oppure ancora in presenza di candidati (sindaco o presidente provinciale o regionale) che riscuotono appeal personale e si configurano di fatto in modo trasversale ai due blocchi contrapposti.

Molti dati sembrano confermare questa interpretazione: in numerose città $i$ sindaci più popolari sono votati indifferentemente da elettori di destra e di sinistra (si pensi al caso di Bassolino a Napoli, di Guazzaloca a Bologna, di Fistarol a Belluno, ecc.); i passaggi di voto tra partiti di centro-destra e partiti di centro-sinistra appaiono sempre straordinariamente limitati; l'indecisione di fondo degli elettori riguarda sempre meno il voto maggioritario rispetto al voto proprozionale.

Dal punto di vista delle scelte elettorali, il quadro che ne esce sembra allora proporre il ritorno ad una nuova stabilità del voto, attraverso la ricostituzione di solide «famiglie politiche». Anche nelle ultime elezioni europee, i rapporti di forza tra centro-sinistra e centro-destra (se sommiamo, indebitamente, tutti i

12 Benché sia tecnicamente improprio, anche nel caso delle consultazioni amministrative possiamo pensare al voto per i sindaci o per i presidenti di Provincia e Regione come una sorta di voto «maggioritario», i voti di lista viceversa potrebbero essere intesi come il voto proporzionale. 
partiti riconducibili a queste due aree) non sono particolarmente mutati, pur in presenza di un elevatissimo livello di cambiamento.

Dal punto di vista del rapporto tra cittadini e politica, infine, l'elemento che emerge in modo più visibile appare il progressivo incremento della sfiducia degli italiani nei propri rappresentanti. Questo scollamento tra classe politica e società, caratteristico del nostro paese, sembra tornare oggi, dopo le aspettative generate dal post-Tangentopoli, a caratterizzare il giudizio di fondo degli italiani sul mondo politico.

La nostra società corre così il rischio che questo dato congiunturale possa divenire cronico, paradigmatico di uno «spirito del tempo» nel quale uno strumento essenziale nella gestione della cosa pubblica viene relegato ai margini dell'immaginario collettivo.

\section{Riferimenti bibliografici}

AA.VV. (1993), Quale mobilità elettorale? Tendenze e modelli. La discussione metodologica sui flussi elettorali, in «Quaderni della Fondazione Feltrinelli», n. 44, Milano, Angeli.

Biorcio, R. e P. Natale (1987), Mobilità e fedeltà elettorale negli anni ottanta. Un'analisi su dati aggregati e di survey, in «Quaderni dell'Osservatorio Elettorale», n. 18, pp. 42-88.

- (1989), La mobilità elettorale degli anni ottanta, in «Rivista Italiana di Scienze Politica», n. 3, pp. 385-430.

Cartocci, R. (1997), Indizi di un inverno precoce: il voto proporzionale tra equilibrio e continuità, in D'Alimonte e Bartolini (1997), pp. 161-205.

Corbetta, P. e A. Parisi (a cura di) (1997), A domanda risponde, Bologna, Il Mulino.

Corbetta, P., A. Parisi e H. Schadee (1988), Elezioni in Italia, Bologna, Il Mulino.

D'Alimonte, R. e S. Bartolini (a cura di) (1997), Maggioritario per caso, Bologna, Il Mulino.

Diamanti, I. e R. Mannheimer (a cura di) (1994), Milano a Roma, Roma, Donzelli.

Francioso, F. e P. Natale (1992), L'elettore tra razionalità e identificazione, in «Quaderni di Sociologia», n. 2, pp. 95-118.

Livolsi, M. (a cura di) (1993), L'Italia che cambia, Firenze, La Nuova Italia.

Mannheimer, R. (a cura di) (1991), La Lega Lombarda, Milano, Feltrinelli. 
Mannheimer, R. e G. Sani (1988), Il mercato elettorale, Bologna, Il Mulino.

Natale, P. (1993), Le tendenze elettorali degli anni Novanta, in Livolsi (1993), pp. 151-181.

- (1994), La nuova mappa geopolitica, in Diamanti e Mannheimer (1994), pp. 85-98.

- (1997), Mutamento e stabilità nel voto degli italiani, in D'Alimonte e Bartolini (1997), pp. 207-235.

- (a cura di) (1998), Abacus. Italia al Macroscopio, Milano, Feltrinelli.

Parisi, A. e G. Pasquino (a cura di) (1977), Continuità e mutamento elettorale in Italia, Bologna, Il Mulino.

Pasquino, G. (1982), Degenerazione dei partiti e riforme istituzionali, Roma-Bari, Laterza.

Pizzorno, A. (1983), Sulla razionalità della scelta democratica, in «Stato e Mercato», n. 7, pp. 3-46.

Ricolfi, L. (1994), Il voto proporzionale e il nuovo spazio politico italiano, in «Rivista Italiana di Scienza Politica», n. 3, pp. 587-630.

Schadee, H. e P. Corbetta (1984), Metodi e modelli di analisi dei dati elettorali, Bologna, Il Mulino.

Vassallo, S. (1997), Struttura della competizione e risultato elettorale, in Corbetta e Parisi (1997), pp. 21-79. 